\title{
Labyrinthe
}

27 | 2007 (2)

La fin des disciplines?

\section{HANNAH ARENDT. Crises de l'État-nation. Pensées alternatives}

Colloque international, 16-18 novembre 2006.

\section{Dario Cecchi}

\section{(2) OpenEdition}

\section{Journals}

Édition électronique

URL : https://journals.openedition.org/labyrinthe/1885

DOI : $10.4000 /$ labyrinthe. 1885

ISSN : 1950-6031

Éditeur

Hermann

\section{Édition imprimée}

Date de publication : 4 juillet 2007

Pagination : 125-130

ISBN : 978-2-9526131-4-9

\section{Référence électronique}

Dario Cecchi, « hanNAh ARENDT. Crises de l'État-nation. Pensées alternatives », Labyrinthe [En ligne], 27 | 2007 (2), mis en ligne le 25 mars 2011, consulté le 26 avril 2022. URL : http://

journals.openedition.org/labyrinthe/1885; DOI : https://doi.org/10.4000/labyrinthe.1885 


\title{
HANNAH ARENDT \\ Crises de l'État-nation. Pensées alternatives
}

\author{
Dario CECCHI \\ dario.cecchi@gmail.com
}

Du 16 au 18 novembre 2006, a eu lieu à l'université Paris-VII un important colloque sur la pensée d'Hannah Arendt, intitulé Crises de l'Étatnation. Pensées alternatives ${ }^{1}$. Il s'est déroulé dans le cadre de la Journée mondiale de la philosophie, promue par l'Unesco, et des célébrations pour le centenaire de la naissance d'Arendt. Le Centre de sociologie des pratiques et des représentations politiques (CSPRP) de l'université ParisVII, qui a organisé la rencontre française, a mis en avant, d'une part, l'intérêt suscité dans la philosophie politique française par la pensée arendtienne, d'autre part, l'élaboration de la pensée d'Arendt précisément durant ses années françaises (1933-1940).

Rappelons deux concepts arendtiens, qui permettront de comprendre mieux certains aspects du colloque: Arendt critique l'idée que la politique passe seulement à travers les formes organisées qui caractérisent la politique moderne - État, institutions, partis. Elle nie l'équation entre violence et pouvoir: le pouvoir ne naît pas du monopole de la violence, comme le veut souvent la philosophie politique. Il est le fruit de la libre association des hommes à travers leurs actions, qui constituent donc le fondement de toute communication politique. Les deux catégories centrales de la pensée arendtienne sont donc celles de la pluralité et de la natalité. La pluralité est le fait que les hommes existent comme individus égaux, en tant qu'êtres humains, mais différents les uns des autres; la natalité désigne la capacité de l'homme de «se révéler» à travers l'action, qui, comme une seconde naissance, lui permet d'accéder au monde commun.

Le colloque avait comme thème général (mais non unique) la crise de l'État-nation. La nation est, dans la conception très critique qui est celle d'Arendt, pensée comme l'ensemble des membres d'une même communauté, unis par des liens de «consanguinité». Cette idéologie moderne

1. Voir http://www.univ-paris-diderot.fr/2006/07-arendt.pdf [consulté le 25 mai 2007]. 
fait ressortir le phénomène de l'antisémitisme, qu'Arendt dissocie de l'antijudaïsme. Le colloque s'est donc développé en oscillant entre ces deux pôles: la question de l'État (et de la souveraineté) et la question de l'antisémitisme comme "explosion» de l'idéologie nationaliste au XIX siècle. Le colloque n'a pas manqué de situer les thèmes dans un cadre philosophique, puisqu'il paraît désormais presque évident que l'on doit considérer Arendt comme une philosophe plutôt que comme une théoricienne de la politique; une penseuse, donc, qui ne s'interroge pas uniquement sur les caractères et les modalités de la participation politique, mais qui enquête sur ses présupposés. Les questions philosophiques posées par la pensée arendtienne étaient au cœur des interventions de chercheurs comme Jacques Taminiaux et Miguel Abensour. Leurs interventions ont clairement montré qu'il existe une dette philosophique d'Arendt à l'égard de Martin Heidegger. On peut aujourd'hui traiter cette question sans faire référence aux événements personnels des deux penseurs ou à l'implication de Heidegger dans le nazisme. Arendt a hérité de Heidegger principalement la méthode et le projet d'une déconstruction de la métaphysique, c'est-à-dire la mise en discussion des hypothèses de la philosophie traditionnelle.

À la fin du colloque, le parcours philosophique d'Arendt a été le thème dominant de la discussion. Étienne Tassin a relu dans une perspective philosophique la critique de la conception de la souveraineté populaire à partir de l'idée de volonté générale: reconsidérer ce problème à travers les analyses d'Arendt revient à se demander à quelles conditions peut se constituer un «sujet» collectif capable d'exercer la faculté de vouloir. Abensour a repris l'interprétation arendtienne du mythe de la caverne de Platon, pour proposer le tableau d'une critique grandiose, par Arendt, de la prétention de souveraineté de la philosophie par rapport à la politique. Tentative particulièrement intéressante pour deux raisons: seule la philosophe féministe italienne Adriana Cavarero s'est confrontée à ce thème de manière spécifique, mais, dans le discours arendtien, Cavarero a privilégié la critique faite à Platon d'avoir dévalué la sensibilité dans l'expérience. Abensour, en revanche, privilégie la question, plus strictement politique, de la représentation philosophique (et plus tard scientifique) de l'action politique; en privilégiant cet aspect, il a le mérite de mettre en évidence la dette d'Arendt envers l'interprétation heideggérienne de Platon, qui est l'un des passages fondamentaux dans la critique de l'incompréhension du concept de vérité par la métaphysique: 
ce qui devint dans la modernité le sujet capable de donner des représentations du monde, voilà, pour Arendt, le sujet souverain.

Déjà Taminiaux, dans l'introduction du colloque, avait ramené Arendt à ses sources philosophiques: à la grande question (heideggérienne) de l'être. Taminiaux a le mérite de reconnaître à Arendt une approche originale, pas totalement dépendante de la philosophie de Heidegger: comme le montre aussi son essai publié dans Critique de la politique. Autour de Miguel Abensour ${ }^{1}$, il essaie de lire Arendt à la lumière de cette phénoménologie française d'orientation levinassienne, qui situe un discours philosophique au-delà de la catégorie de l'être. Permettonsnous d'exprimer un désaccord sur ce point, et de formuler en même temps une première critique concernant ce beau colloque.

Il est dommage qu'ait manqué la comparaison avec les disciplines historiographiques. Était prévue la participation du grand historien Pierre Vidal-Naquet qui avait étudié le rapport entre Arendt et la Shoah. VidalNaquet a malheureusement disparu peu avant le colloque. La première session a donc été dédiée à sa mémoire, et Katrin Tenenbaum, de l'université La Sapienza de Rome, s'est attardée sur la possibilité de retrouver en Arendt un concept de peuple et en particulier du peuple juif: la chercheuse italienne nous a ramenés à une question encore ouverte dans la pensée d'Arendt, celle de son rapport avec le sionisme. Il s'agit d'un thème «brûlant», si nous pensons à l'accusation d'antisionisme - voire d'antisémitisme - que certaines de ses prises de position lui ont value. Or, si l'on prend en compte la recherche de Tenenbaum, qui est encore en cours, on arrive à la conclusion suivante, encore implicite dans son intervention: contrairement à ce que voudrait une certaine vulgate, Arendt n'a jamais été antisioniste. Tout au plus avait-elle un point de vue extrêmement «laïque» sur la question nationale juive. La question permet de mettre à l'épreuve la validité de l'approche arendtienne de la politique, selon laquelle il n'est pas d'intérêts, de valeurs, de principes qui doivent entrer dans la politique, mais seulement les règles d'une participation active à la vie politique de sa propre communauté, dans le cadre d'un certain élitisme politique.

Martine Leibovici, chercheuse française qui a remis la question juive au centre des recherches sur Arendt, s'est interrogée sur la manière qu'a la penseuse de distinguer différentes formes d'antisémitisme, en les met- 
tant en relation avec la genèse de différents nationalismes européens. Sur ce point, l'absence d'un historien comme Vidal-Naquet a été plus sensible encore: il nous semble en effet qu'a aussi été esquivée, au cours de ce colloque, une question méthodologique importante. Arendt, intellectuelle connue pour ses analyses éclairantes de la modernité et pour sa réflexion extrêmement originale, a exprimé dans des textes comme Les Origines du totalitarisme ou Essai sur la révolution des thèses historiographiquement controversées à propos d'événements centraux pour l'époque moderne et contemporaine: essayer de réfléchir aux œuvres d'Arendt en mettant en œuvre une méthodologie plus strictement historiographique aurait eu le mérite de repenser certaines de ses affirmations, de mieux définir la validité et le champ d'action de certains de ses concepts à la fois brillants et contestés.

Parmi les jeunes chercheurs qui sont intervenus dans la session de l'après-midi du 16 novembre, Valérie Gérard, de l'université Lille-III, a parlé du concept d'«exceptionnalité» dans la pensée arendtienne (on sait avec Carl Schmitt que l'exceptionnalité est l'une des catégories remarquables de la souveraineté). Dana Villa, spécialiste important d'Arendt - quoique jeune -, est aussi intervenu. Déjà connu pour ses recherches sur le rapport entre Arendt et Heidegger (si importantes que certains distinguent entre deux lignes de pensée, la sienne et celle de Taminiaux), Villa a proposé une confrontation entre l'Arendt des Origines du totalitarisme et l'Adorno (il serait plus juste de dire: Adorno et Horkheimer) de la Dialectique de la raison. Le parcours de Villa, déjà présenté à un colloque qui a eu lieu en 2005 à Bari, en Italie, ne paraît pas encore pleinement convaincant; reste l'extrême intérêt que présente une confrontation entre ces deux grandes narrations de la modernité. La première session du 17 novembre partage avec cette dernière un thème, pas complètement explicite: le problème de la souveraineté dans la pensée d'Arendt.

Les communications du 17 novembre mettent en évidence la critique adressée par Arendt à une politique dont le fondement serait une conception de la souveraineté, en particulier quand, dans l'histoire européenne, les États souverains deviennent États nationaux impérialistes: Anne Amiel, par exemple, traite la pensée d'Arendt comme une science politique, arrivant à la considérer comme une «non-philosophie». Sa thèse, soutenue avec lucidité et intelligence, reste provocatrice: comment parler d'une politique faite de domination, de violence et de guerre dans le cadre d'une science politique qui dénie à toutes ces conceptions leur 
«caractère politique»? Sonia Dayan-Herzbrun a évoqué l'Afrique comme théâtre de la rencontre entre la «civilisation» européenne et le «sauvage», ne manquant pas de mettre en évidence certaines limites d'Arendt à la compréhension de la question africaine (et afro-américaine). Wolfgang Heuer et Danièle Lochak se sont concentrés sur le problème des minorités dans le contexte d'un État souverain national: pour Heuer, elles doivent avoir un «caractère politique», tandis que pour Lochak elles constituent une force antagoniste «non-nationale» à opposer à la souveraineté de l'État-nation.

Le dernier jour, le thème de la souveraineté a été traité de manière plus théorique par les élèves d'Arendt, Elizabeth Young-Bruehl et Jerome Kohn, qui ont confronté leurs approches au cours d'un dialogue public. Kohn a reconstruit la généalogie du concept de souveraineté à travers les plus grands penseurs politiques des temps modernes pour arriver à la critique arendtienne de cette conception. Young-Bruehl a repris le thème, en proposant une interprétation de la pensée d'Arendt comme un «néo-républicanisme». Selon elle, l'important est de comprendre comment, de l'œuvre posthume d'Arendt, La Vie de l'esprit, est sortie, à l'état d'ébauche, l'idée d'une «république de l'esprit», c'est-à-dire d'une harmonie entre les différentes facultés mentales dans le but de produire un rapport cohérent avec la réalité qui nous entoure: autrement dit, YoungBruehl a repris la pensée plus philosophique de la dernière Arendt, en essayant de lui attribuer la systématicité qui lui manque à cause de la mort d'Arendt, qui n'a pas terminé La Vie de l' esprit. La proposition de YoungBruehl n'est pas totalement convaincante: elle fait courir le risque de ramener la pensée d'Arendt à une erreur métaphysique connue, consistant à prendre la réalité de l'esprit pour la réalité de l'expérience. YoungBruehl, comme du reste les autres interventions plus philosophiques, n'a pas parlé de l'interprétation arendtienne de la Critique de la faculté de juger de Kant, qui est selon nous la référence philosophique d'Arendt pour penser le rapport entre réalité et pensée. Kohn a conclu le dialogue en remettant en discussion le concept de démocratie des conseils cher à Arendt, dépouillé de ses coloris marxiens (auxquels Arendt, au demeurant, n'a jamais adhéré), pour le ramener à une critique de l'actualité politique américaine et au problème, déjà dénoncé par Arendt, de la perte de participation politique aux États-Unis.

Au cours de ce colloque, de multiples thèmes de la pensée arendtienne ont été parcourus et reliés à un sujet, le problème de l'État-nation; cer- 
tains aspects des travaux en cours, comme la question juive, ont été mis en valeur sans faire l'objet d'une recherche pédante; un fort encadrement philosophique a été donné à la discussion des thèses arendtiennes, et on a reconnu en Arendt une philosophe authentique, qui tient aux questions non immédiatement politiques. Sur ce dernier point, néanmoins, permettons-nous d'avancer une autre critique.

Trop peu d'importance a été donnée à l'apparition d'une ligne philosophique autonome chez Arendt: selon nous, se pose chez elle la question de la possibilité d'exercer la pensée (et donc de faire de la philosophie) sans jamais abandonner la condition de l'être humain, appartenant à un monde de relations complexes et continuellement renouvelables, que l'on ne peut jamais ramener «une fois pour toutes » aux idées ou aux concepts philosophiques. Cette ligne, pour Arendt, part de Socrate pour arriver, dans la modernité, jusqu'à Kant. Socrate et Kant nous ont paru les grands absents de la féconde discussion du colloque: à travers eux, on peut penser une critique de l'État-nation, qui donne l'exemple d'un autre espace public, celui de l'échange des idées. La pensée d'Arendt est peutêtre une pensée du «différent de», et pas, comme celle d'Emmanuel Levinas, une pensée de l'«autrement que». Autrement dit, il semble peu utile d'enfermer la pensée d'Arendt dans la philosophie comme dans une réserve indienne: en face d'une politique qui peut aujourd'hui encore, à certains égards, sembler «souveraine» et violente, est-ce effectivement une solution que d'enfermer ces pensées critiques dans les confins étroits de leurs présupposés philosophiques? Ce colloque a offert la possibilité de repenser le rôle de ces grandes réflexions qui ont caractérisé le second après-guerre: les utiliser implicitement comme instruments de critique, qui ne trouvent pas une correspondance directe dans l'action politique, qui au contraire préfigurent peut-être déjà dans la critique même une forme d'action qui éduque à voir le monde différemment. Si l'on ne prend pas cela comme un slogan facile, il est nécessaire de passer ces réflexions à un crible critique et méthodologique particulièrement fin, pour en éprouver la solidité et la capacité effective d'interpréter la réalité.

Les prochains colloques sur Arendt devront s'interroger toujours davantage sur le rapport entre réflexion et historiographie, et sur la validité de certaines réflexions à la lumière de l'économie, du développement technologique effréné et de la globalisation.

Traduit de l'italien par Patrizia Rafat 